\title{
A Novel Urine Method for the Diagnosis of Active Tuberculosis by Immunoassay for the Detection of ESAT-6 Using Hydrogel Nanoparticles in HIV Patients
}

\author{
Luz Huaroto1,2, Raquel Mugruza1 ${ }^{*}$, Marinelly Roca1, Ricardo Antiparra ${ }^{3}$, César Ticona1, \\ Patricia Sheen ${ }^{3}$, Eduardo Ticona1,2, Robert H. Gillman' ${ }^{4}$, Alessandra Luchini ${ }^{5}$, Lance Liotta ${ }^{5}$ \\ ${ }^{1}$ Major National University of San Marcos, Lima, Peru \\ ${ }^{2}$ Dos de Mayo National Hospital, Lima, Peru \\ ${ }^{3}$ Universidad Peruana Cayetano Heredia, Lima, Peru \\ ${ }^{4}$ Johns Hopkins, Bloomerg School of Public Health, Baltimore, USA \\ ${ }^{5}$ Center for Applied Proteomic and Molecular Medicine, George Mason University, North Virginia, USA \\ Email: ^raquel_mugruzapineda@yahoo.com.pe
}

How to cite this paper: Huaroto, L., Mugruza, R., Roca, M., Antiparra, R., Ticona, C., Sheen, P., Ticona, E., Gillman, R.H., Luchini, A. and Liotta, L. (2021) A Novel Urine Method for the Diagnosis of Active Tuberculosis by Immunoassay for the Detection of ESAT-6 Using Hydrogel Nanoparticles in HIV Patients. Journal of Tuberculosis Research, 9, 73-84. https://doi.org/10.4236/jtr.2021.92006

Received: January 13, 2021

Accepted: April 22, 2021

Published: April 25, 2021

Copyright ( 2021 by author(s) and Scientific Research Publishing Inc. This work is licensed under the Creative Commons Attribution International License (CC BY 4.0).

http://creativecommons.org/licenses/by/4.0/ (c) (i) Open Access

\begin{abstract}
Background: In patients with HIV, conventional tests are of low sensitivity; therefore, a new diagnostic test with hydrogel nanoparticles with reactive blue dye is proposed, which allows the capture, conservation and concentration of ESAT-6 in urine. NIPAs are copolymers that capture low molecular weight proteins and protect against enzymatic degradation. Using an immunoassay, it is possible to detect and quantify ESAT-6 present in urine as a possible marker of active TB. Design/Methods: Study in Lima, Peru, HIV+ participants, $\geq 18$ years with and without tuberculosis (TB). Smear microscopy, culture in solid medium and urine immunoassay were performed. The reference was the diagnosis of TB by radiological or clinical microbiological criteria (indication for TB treatment). There were 2 preanalytical processes: untreated and treated urine (centrifuged, heated), then incubation with NIPAm. After washing, elution, sonication, heat and centrifugation, the final eluate was obtained. This was placed on nitrocellulose membranes, which by means of fixation and incubation processes with anti-ESAT- 6 and anti-IgG antibodies, revelation with C-DiGit ${ }^{\oplus}$ Blot Scanner and FluorChem R FR0001. Calibration curves were included in the membranes, density was measured using Image $\mathrm{J}$ software. ROC curves, sensitivity and specificity were obtained. Results: The result by groups was HIV+ patient: ROC: 0.75 , cut-off point $\geq 24.06 \mathrm{ng} / \mathrm{ml}$, sensitivity $76.32 \%$, specificity $68.89 \%$, patients $\leq 200$ cells CD $4 \mathrm{~mm}^{3} / \mathrm{ml}$, ROC: 0.78 , cutoff point $\geq 26.20 \mathrm{ng} / \mathrm{ml}$, sensitivity $75.86 \%$, specificity $71.88 \%$, pa-
\end{abstract}


tients $>200 \mathrm{CD} 4 \mathrm{~mm}^{3}$ cells/ml, ROC: 0.73 , cutoff point $\geq 24.6 \mathrm{CD} 4 \mathrm{~mm}^{3} / \mathrm{ml}$, sensitivity $73.68 \%$, specificity $73.68 \%$. Conclusions: The ESAT-6 detection assay using NIPAm was effective, with higher rates in patients with $\leq 200$ CD4 cells $/ \mathrm{mm}^{3}$, the test being more sensitive than smear and culture, but less specific.

\section{Keywords}

Tuberculosis, HIV+, Hydrogel Nanoparticles and ESAT-6

\section{Introduction}

Currently, tuberculosis (TB) and human immunodeficiency virus (HIV) infection are two of the leading causes of infectious mortality worldwide [1]. Approximately $25 \%$ of the world's population are infected with TB and at possible risk of active disease. In 2018, the World Health Organization (WHO) reported 10 million new TB cases, of which $8.6 \%$ also had HIV infection [2], approximately 251,000 deaths. In Peru, it is the main cause of death in the HIV patients TB [3] [4] [5].

Patients HIV positive are highly susceptible to tuberculosis infection, due to their compromised immune system, and therefore low immune response, which does not form caverns, therefore, his patients had a decreased proliferation of bacilli, that difficult the conventional test like direct bacilli observation, culture and nucleic acid amplification [6] [7] [8]. This is the main reason for late diagnosis and treatment initiation, increasing the probability of M. Tuberculosis spread to other organs.

Currently, in low and middle countries the diagnosis of TB in HIV population is based on smear ZN staining, a test with low sensitivity $28 \%$, cultures (Ogawa, MODS and Bactec) have sensitivity greater than $68 \%$, however takes a long incubation period and need high biosecurity measures [9] [10] [11] [12]. Molecular tests such as Xpert MTB/RIF 79\%, genotype 30\% are very effective, but high cost, also, are aimed more to determine drug sensitivity than diagnosis. Therefore, its implementation and use in low complexity laboratories is not very feasible [13] [14]. Radiology diagnosis is not very effective in HIV patients because they don't present the conventional radiologic patrons.

Several studies are searching active TB markers in other samples than sputum, focus on M. tuberculosis antigens detection in urine, due to its easy sample collection, less risk of aerosols for laboratory personnel and lower cost per application and use in a low complexity laboratory. However, the enzymatic degradation of antigens in urine doesn't allow the antigen detection [15].

Other tests have been developed, such as the lipoarabinomannan detection (LAM) in urine by immunochromatography, which is applied in patients with HIV infection with $<200 \mathrm{CD} 4 \mathrm{~mm}^{3}$ cells with a sensitivity of $4.0 \%$ and up to $66.7 \%$ in $<50 \mathrm{CD} 4 \mathrm{~mm}^{3}$ cells [12]. Due to this, its use is diminished and mainly 
applicable to diagnosis disseminated $\mathrm{TB}$, a very advanced stage of tuberculosis infection [7] [8] [16] [17]. Thus, it is necessary to develop diagnostic tests that aren't based on the detection or isolation off bacillus in sputum, but on the detection of Mycobacterium antigens that are present in an easily obtained sample such as urine. Esat- 6 antigen has been investigated to have greater sensitivity and specificity [7] [18] [19] [20]. However, its use in a diagnostic test has not yet been accomplished.

Hydrogel nanoparticles (NIPAm) have been applied, which are three-dimensional hydrophilic copolymers of $\mathrm{N}$-isopropylacrylamide (pNIPAm) [2] that, by size exclusion and affinity chromatography in solution, capture low molecular weight proteins, concentrate and protect of endogenous enzymatic degradation. This method of using nanoparticles to capture antigens in infectious diseases has been used as congenital Chagas disease, showing promising results. In vitro studies carried out by George Mason University (USA) report a 50-fold increase in the detection capacity of ESAT-6, CFP10 and LAM by SDS PAGE compared to the normal value [21] [22] [23] [24] [25]. Hydrogel nanoparticles made on a reactive blue base (NIPAm/rb) have been shown to be the most efficient and best captured ESAT-6 antigens in Western blot analysis.

Due to the need to develop new simple, fast and easy-to-implement diagnostic tests, with greater sensitivity and specificity a new method is with the use of hydrogel nanoparticles for the determination of ESAT antigen-6. Profiling as one of the easiest to apply due to its ease in obtaining the sample and the possible greater sensitivity than other tests.

For these reasons, the main objective of this study is to determine a diagnostic method for active TB in patients with HIV co-infection, through the detection of ESAT-6.

\section{Materials and Methods}

\subsection{Study Design and Population}

Experimental study of the diagnostic test, carried out in Lima, participants from Dos de Mayo National Hospital and 7 peripheral centers belonging to 4 study groups: group $1(\mathrm{~TB}+, \mathrm{HIV}+), 2$ (TB-, HIV+), 3 (TB+, HIV-) and 4 (TB-, HIV-), $\geq 18$ years and capable of produce a sputum sample. TB+ status was defined as active TB from any case with less than 3 days of TB treatment and HIV+ status was by documented HIV infection. Enrollment was carried out in 2 years (02/2017-02/2019). Sputum samples were collected and a random non-sterile urine sample with mínimum volume $90 \mathrm{ml}$.

All sputums had smear and culture in Ogawa; MODS in some cases (When there was a enought sputum sample volume); immunoassay for ESAT-6 with NIPAm in urine and rapid HIV test (where applicable). Likewise, a socioeconomic questionnaire, TB contact, medical history, concomitant treatment, as well as signs and symptoms of TB were applied. Drug susceptibility testing of standard care was recorded. 
The diagnosis of TB was made by 3 criteria: microbiological, positivity to smear, Ogawa or MODS culture, hain testin sputum and other samples; radiological, patterns compatible with active tuberculosis infection and indication of immediate; and clinical TB treatment, determined by signs and symptoms compatible and indication of immediate TB treatment. HIV+ patients were followed up for 1 year, and those who later presented TB were group reassigned. Follow-up was carried out by patient self-report if they had active tuberculosis in the previous year.

The samples were processed in two laboratories: Center for Applied Proteomics and Molecular Medicine, at George Mason University (U. Mason) and at the Biochemistry and Informatics Laboratory, Research and Development Laboratories, Universidad Peruana Cayetano Heredia (UPCH). Test standardization processes were carried out in both laboratory.

\subsection{Experiment}

A urine sample was collected, transported in $2^{\circ} \mathrm{C}-8^{\circ} \mathrm{C}$. There were 2 preanalitycal procedures for treated and untreated urine 1) Untreated urine, directly incubated with NIPAm (45/1), incubated at room temperature for 30 minutes and centrifuged at $4000 \mathrm{~g}$ for 20 minutes, the pellet resuspended in $1 \mathrm{ml}$ of urine, aliquoted and stored at $-80^{\circ}$ C. 2). Treated urine, immediately frozen at $-80^{\circ} \mathrm{C}$, preserve and in laboratory conditions heated to $100^{\circ} \mathrm{C}$ in drie heat for $10 \mathrm{mi}$ nutes, centrifuged at $16,000 \mathrm{rpm}$ for 15 minutes. A $1 \mathrm{ml}$ aliquot of supernatant is recovered, and incubated with $200 \mu \mathrm{l}$ of NIPAm at room temperature and subsequently, centrifuged at $16,000 \mathrm{rpm} / \mathrm{min}$ with the aim of obtaining the complex ESAT-6 + Nipam pellets. In the untreated urine samples, the Nipam vol was recorded to adjust the ESAT- 6 concentration, this factor allowed the samples to be compared with the calibration curve.

Calibration curve, included in each immunoassay, with concentrations of recombinant antigen ESAT-6 (abcam, ab124574) and a negative control splashed into $1 \mathrm{ml}$ of urine were included. In treated urine, concentrations of $100 \mathrm{ng} / \mathrm{ml}$, $50 \mathrm{ng} / \mathrm{ml}, 25 \mathrm{ng} / \mathrm{ml}$ and $0 \mathrm{ng} / \mathrm{ml}$ were included and in untreated urine, 100 $\mathrm{ng} / \mathrm{ml}, 40 \mathrm{ng} / \mathrm{ml}, 10 \mathrm{ng} / \mathrm{ml}$ and $0 \mathrm{ng} / \mathrm{ml}$. Depending on the type of preanalityc (treated and untreated urine), the curve passes the same preanalytical procedures.

Elution, the pellets (Nipam + low molecular weight proteins) were washed 3 times with $\mathrm{H}_{2} \mathrm{O}$ Milli-Q, centrifuged at $16,000 \mathrm{rpm}$ for 15 minutes. The pellet was eluted with $50 \mu \mathrm{l}$ of Novex ${ }^{\mathrm{TM}}$ Tris-Glycine SDS Sample Buffer $(2 \times)(5 \%$ 2-Mercaptoethanol, CAS Number 60-24-2), subsequent sonication for $30 \mathrm{mi}$ nutes at room temperature, heated to $100^{\circ} \mathrm{C}$ for 5 minutes and centrifuged at $16,000 \mathrm{rpm} / \mathrm{min}$.

The supernatant passed the $\mathrm{HiPPR}^{\mathrm{rm}}$ Detergent Removal Spin Column Kit columns according to the manufacturer's instructions, obtaining the detergent-free eluate for immunoassay.

Immunoassay, an immunoblotting membrane (IMMUN BLOT PVDF MEM, 
1620176, Biorad) was hydrated with 1 alcohol bath and 3 water for 1 minute each. To the hydrated membrane, the calibration curve and the samples were included in spots, two rounds of $1 \mu$ l each.

Incubation was performed by rotation at room temperature ( 1 - 3 hours) and in refrigeration for long periods ( 4 - 12 hours). The times and dilutions varied according to the procedure of each laboratory.

The membrane was incubated with blocking buffer (phosphate buffer saline (PBS), 0.2\% I-block), incubation time 1.5 - 12 hours (U. Mason) or 2 hours (UPCH). Subsequent incubation with anti-ESAT-6 monoclonal antibody (abcam, ab26246) diluted in blocking buffer, dilution 1/250, for 1.5 - 12 hours (U.Mason) or $1 / 1000$, for 3 hours (UPCH). 3 washes with PBS-T (PBS, $0.1 \%$ tween) for 15 minutes each.

The next incubation is with horseradish peroxidase-labeled goat polyclonal secondary antibody (Invitrogen A16084), dilution 1/5000 with incubation time for 2 hours (U. Mason) and for 1 hour (UPCH). Consecutively, 3 washes with PBS-T of 15 minutes each were carried out.

Then, the development is with radish peroxidase (HRP) substrate of enhanced chemiluminescence based on luminol (ECL) for 5 minutes away from light.

Two chemiluminescence systems were used, in Mason, FluorChem R FR0001; $\mathrm{UPCH}, \mathrm{C}$ Digit Blot Scanner. Photographs were taken of the membrane without a filter with a light exposure of 30 seconds and 3 minutes, the photograph with the best resolution, the lowest background, and the highest reactivity in the positive controls was chosen.

Reveled, spot intensity was measured with Image J NIH software. A relationship between calibration curve and their image intensity was established. Using this equation, the simple concentración was calculated.

In some membranes with an unclear and therefore invalid calibration curve, the equation of a valid standard curve of a membrane performed on the same day (same eluates of the calibration curve) was used.

\subsection{Analysis Plan}

Statistical software Stata 13 was used. The relationship equation between the spots densities and the curve concentration was performed by linear regression. ROC (Receiver operating characteristic) curves were used to determine sensitivity and specificity of different cut-off points for the concentration of ESAT- 6 in urine for the diagnosis of TB in the HIV population, having as a reference test, positivity at least one of the microbiological criteria, clinical or radiological. Comparisons were made between diagnostic and reference tests, and I also made comparisons between each test. Likewise, subgroups of patients were analyzed regarding pulmonary and extrapulmonary TB status, as well as CD4 level, and the sensitivity and specificity in each subgroup were calculated. The statistical power of the sample size was $89.90 \%$ with a significance level of 0.05 , using an independent group comparison and expected sensitivity of the test of $60 \%$ com- 
pared to the $30 \%$ smear test.

\subsection{Ethical Considerations}

The protocol was approved by the Ethics Committee in Biomedical Research of the Dos de Mayo National Hospital and reviewed by the Ethics Committee of the Universidad Peruana Cayetano Heredia in December 2015.

\section{Results}

332 patients were evaluated, in four study groups 1 (HIV $+\mathrm{TB}+)(59), 2$ (HIV+ TB-) (91), 3 (HIV- TB+) (92) and 4 (HIV- TB-) (90). There was a predominance of males $(89.83 \%, 82.61 \%, 63.04 \%, 44.44 \%)$, the median age and interquartile range of age was similar, being in the range of 25 - 35 years (Table 1 ).

The ESAT- 6 protein in urine was present in all study groups, with TB patients having a higher concentration. Group 1 presented a median and interquartile range of $38.51 \pm 46.98 \mathrm{ng} / \mathrm{ml}$; group $3,28.33 \pm 25.56 \mathrm{ng} / \mathrm{ml}$. The TB-control patients, group $214.06 \pm 31.17 \mathrm{ng} / \mathrm{ml}$ and finally group $49.16 \pm 27.98 \mathrm{ng} / \mathrm{ml}$.

Regarding TB patients, within groups 1 and 3, there was a higher proportion of patients with pulmonary TB, 42 (71.19\%) and 86 (93.48\%), respectively. Of these, $20(33.90 \%)$ and $57(61.96 \%)$ showed positivity in smear microscopy, 26 (45.61) and 76 (83.52) in Ogawa culture and 15 (41.67) and 68 (74.73) in MODS culture. Sensitivity to drugs was only determined in 23 patients $(38.98 \%)$ in the HIV+ participants' and in 78 participants (84.78\%) in the HIV-group.

The median ESAT-6 concentration in urine has a statistically significant association with TB status $(\mathrm{p}=0.00)$. The median concentration of ESAT- 6 in urine did not show statistical association with the level of HIV viral load copies/ml ( $p$ $=0.45)$ and CD4 cells $/ \mathrm{ml}(\mathrm{p}=0.26)$ in the HIV population (Table 2).

Table 1. Sociodemographic data.

\begin{tabular}{lcccc}
\hline & \multicolumn{3}{c}{ Grupo } \\
\cline { 2 - 4 } & VIH+ TB+(59) & VIH+ TB- (91) & VIH- TB+ (92) & VIH- TB- (90) \\
\hline Sex & & & \\
Male & $53(89.83)$ & $76(82.61)$ & $58(63.04)$ & $40(44.44)$ \\
Age & $33 \pm 15$ & $35 \pm 15$ & $29.5 \pm 22.5$ & $25 \pm 15$ \\
Ocupation & $4(6.77)$ & $7(7.69)$ & $15(16.67)$ & $46(51.68)$ \\
Student & $1(1.69)$ & $3(3.30)$ & $0(0.00)$ & $19(21.35)$ \\
Health Personnel & $46(76.24)$ & $68(74.73)$ & $65(72.21)$ & $20(20.22)$ \\
Employed & $9(15.25)$ & $13(14.29)$ & $10(11.11)$ & $4(4.49)$ \\
Unemployed & & & & \\
History of TB & $19(32.76)$ & $22(24.18)$ & $12(13.33)$ & $4(4.60)$ \\
Yes & $38.51 \pm 46.98$ & $14.06 \pm 31.17$ & $28.33 \pm 25.56$ & $9.16 \pm 27.98$ \\
ESAT-6 (ng/ml) & & & & \\
\hline
\end{tabular}

*Median and interquartile range. 
Table 2. HIV and TB status.

\begin{tabular}{cccc}
\hline & VIH $+\mathrm{TB}+(59)^{*}$ & $\mathrm{VIH}+\mathrm{TB}-(91)^{*}$ & $\mathrm{p}^{* *}$ \\
\hline CD4/mm ${ }^{3}$ cell level & $105 \pm 214$ & $311 \pm 321$ & 0.00 \\
HIV viral load copies/mL & $112,250 \pm 396,800$ & $403 \pm 128,500$ & 0.00 \\
ESAT-6 $\mathrm{ng} / \mathrm{ml}$ & $38.51 \pm 46.98$ & $14.06 \pm 31.17$ & 0.00 \\
\hline
\end{tabular}

${ }^{*}$ Median and interquartile range, ${ }^{* *}$ Mann Whitney $\mathrm{U}$ test.

Table 3. Sensitivity and specificity of the ESAT detection test using NIPAm according to subgroups.

\begin{tabular}{lcccccc}
\hline & ROC & $71.72 \%$ & Obs & 147 & IC & $0.63-0.80$ \\
TB all locations & Cutpoint & $\geq 24.06$ & Sensitivity & $71.92 \%$ & Specificity & $68.89 \%$ \\
& Cutpoint & $\geq 37.17$ & Sensitivity & $52.63 \%$ & Specificity & $81.11 \%$ \\
\hline $\begin{array}{l}\text { Patients with HIV } \\
\text { infection <200 CD4 }\end{array}$ & ROC & $73.51 \%$ & obs & 76 & IC & $0.61-0.85$ \\
cells-TB all locations & Cutpoint & $\geq 21.78$ & Sensitivity & $79.55 \%$ & Specificity & $68.75 \%$ \\
& Cutpoint & $\geq 26.2$ & Sensitivity & $70.45 \%$ & Specificity & $71.88 \%$ \\
$\begin{array}{l}\text { Patients with HIV } \\
\text { infection }>200 \text { CD4 } \\
\text { cells-TB all locations }\end{array}$ & ROC & $70.28 \%$ & obs & 83 & IC & $0.58-0.83$ \\
\hline & Cutpoint & $\geq 24.6$ & Sensitivity & $72.00 \%$ & Specificity & $68.97 \%$ \\
\hline $\begin{array}{l}\text { Pulmonary TB } \\
\text { ROC }\end{array}$ & $75.15 \%$ & obs & 128 & IC & $0.66-0.84$ \\
& Cutpoint & $\geq 21.78$ & Sensitivity & $78.95 \%$ & Specificity & $65.56 \%$ \\
\hline
\end{tabular}

A sensitivity and specificity analysis of the test was performed according to study subgroups. Thus, for the diagnosis of TB all locations in patients with HIV infection, an AUC of 71.72, sensitivity of $71.92 \%$, specificity of 68.89 was determined, considering as a cut-off point $\geq 24.6 \mathrm{ng}$ ESAT- $6 / \mathrm{ml}$ of urine (Table 3 ).

When the population was divided into patients with and without AIDS phase $(<200 \mathrm{CD} 4$ cells/ml). In patients with a CD4 cell level $<200$ cells/ml, an AUC 73.51 , sensitivity $70.45 \%$, specificity $71.88 \%$ were determined considering a cut-off point $\geq 26.20 \mathrm{ng}$ ESAT-6/ml of urine, considering 76 samples for analysis. In the group with CD4 cells $>200$ cells/ml, AUC 70.28, sensitivity $72.00 \%$, specificity $68.97 \%$ considering a cut-off point $\geq 24.60 \mathrm{ng}$ ESAT-6/ml of urine, with 83 samples for analysis.

For the diagnosis of pulmonary TB, independent of CD4 cells, an AUC 75.15, sensitivity $78.95 \%$ and specificity of $68.89 \%$ were determined, with a cut-off point $\geq 24.6 \mathrm{ng}$ ESAT-6/ml of urine, considering 128 samples to be analyzed.

\section{Conclusions}

For the study groups, AUC value was greater $>70$, sensitivity $>60 \%$ and specificity $>60 \%$ were found. The group with the best rates was for the diagnosis of pulmonary tuberculosis in patients with HIV infection.

Urine samples are easy and quicker to obtain, is only necessary refrigeration 
as soon as possible. Is plausible method, with lower risk of handling potentially infectious samples. Likewise, by detection of a TB marker, decreases high exposure to health personnel by obtaining and managing samples like bronchial aspirates, biopsies, or induced in HIV patients.

In the study, two preanalytical procedures were analyzed for treated and untreated urine both calibration curves were validated. However, there was no significant difference between the calibration curves of both methods. The untreated urine protocol requires fewer preanalytical conditions and is more feasible for field application. However, in recent years, health centers have more equipment, making it possible to use a thermoblock and a centrifuge, where samples can be processed into pellets for transport in the laboratory. Although the nanoparticles are stable at room temperature and could be used in the field, the protocol for treated urine and its use in the laboratory allows us to include the initial solution (urine) and the supernatant in the immunoassay, which serves as an additional internal control of the test.

In the study, the development of the immunoassay was carried out in two equipment, the method was validated in both FluorChem R FR0001 and C Digit Blot Scanner equipment, however, a difference between the thresholds was detected. For the application of the test, the method must be validated using the available chemiluminescence reader.

The immunoassay procedure takes up to 12 hours, despite the duration of the procedure, it is considerably less than an incubation time of a culture and at a lower cost of a molecular test. More experiments are necessary to decrease the time in the procedures in order for the method to be more adaptable to the clinical field.

The sensitivity and specificity values of the NIPA ESAT- 6 urine test are greater than $70 \%$, more sensitive, less specific than smear microscopy (sensitivity $28 \%$, specificity $92 \%$ ) and less sensitive or specific than a culture (sensitivity $80 \%$ - 90\%, specificity 98\%) in the HIV population, all with an AUC value $>73 \%$, classifying it as a good test.

Several studies have tested the detection of new biomarkers in both sputum and urine, thus, in 2017, a study testing a cocktail immunoassay of antigens such as ESAT-6, CFP10 and MPT64 in urine, the sensitivities were low $68 \%, 2 \%, 22 \%$ and $31.6 \%$, respectively [26].

In Indonesia, they carried out a qualitative detection study of antigens by immunochromatography such as ESAT-6, CFP-10 and MPT64, a 78\% specificity and $68.8 \%$ sensitivity were determined, determining that the markers should more study [2].

In Geneva, performed a new electrochemiluminescence method for the detection of ESAT- 6 in urine and serum, the method presented a minimum detection threshold as $\mathrm{pg} / \mathrm{ml}$. In urine, the sensitivity of LAM and ESAT- 6 were $93 \%$ and $65 \%$, respectively. For ESAT-6, 55\% and 46\% were presented, respectively [27].

Likewise, there is the Silva LAMP, for the detection of LAM for the diagnosis of extrapulmonary tuberculosis, which determined a sensitivity of $60 \%$ for Pul- 
monary TB and $67 \%$ for Extrapulmonary TB. Although this test has presented good results, its validation is still under study. Various studies have studied the ability to detect ESAT-6 in urine and blood by different immunoassay methods, however, the sensitivity remains low. There is the possibility that the low levels of sensitivity are due to the low concentration in urine or the enzymatic degradation [28] [29] [30].

Due to this, in 2013, an immunoassay with gold nanoparticles, an immunoassay (ELISA) was carried out for the detection of ESAT-6 where promising results were presented, this type of nanoparticles presented a concentration factor of $7.5 \times$. However, the hydrogel nanoparticles showed greater stability and a concentration factor of up to $50 \times$ [21].

The study method determined to be applicable for the diagnosis of TB in all locations, however, better rates were determined for the diagnosis of pulmonary $\mathrm{TB}$, this possibly due to misclassification bias in patients with extrapulmonary TB due to the low sensitivity of the tests for the TB diagnosis and clinical assessment for initiation of treatment. To determine the sensitivity and specificity of the extrapulmonary TB test, confirmatory studies by molecular or microbiological biology with more sensitive methods are necessary, as well as a larger sample size. However, the results of the study allow us to say that this test is possibly even more sensitive than current conventional tests. There are molecular tests that have determined a sensitivity level greater than $40 \%$ in the non-HIV population. However, in a population with coinfection, it may have a lower level. As well as the difficulty of applying mPCR tests [25].

The present study of nanoparticles shows promising results of sensitivity, the test can be used as a TB screening that must be interpreted together with a clinical and epidemiological evaluation, thus reducing the delay in the start of treatment. However, larger studies of latent TB should be performed in the same population to eliminate possible classification bias.

\section{Funding}

Study is financed by the Basic and Applied Sciences Project Contest of FONDECYT-Lima, Peru (Contract 120-2016).

\section{Conflicts of Interest}

The authors declare no conflicts of interest.

\section{References}

[1] World Health Organization (2020) Informe mundial sobre la tuberculosis. https://www.who.int/teams/global-tuberculosis-programme/tb-reports

[2] Organización Panamericana de la Salud (2017) Coinfeccion TB/VIH: GuíaClínica Regional. Actualización 2017.

[3] Mendelson, M. (2007) Diagnosing Tuberculosis in HIV-Infected Patients: Challenges and Future Prospects. British Medical Bulletin, 81-82, 149-165. https://doi.org/10.1093/bmb/ldm009 
[4] Kaforou, M., Wright, V.J., Oni, T., French, N., Anderson, S.T., Bangani, N., et al. (2013) Detection of Tuberculosis in HIV-Infected and -Uninfected African Adults Using Whole Blood RNA Expression Signatures: A Case-Control Study. PLOS Medicine, 10, e1001538. https://doi.org/10.1371/journal.pmed.1001538

[5] Richeldi, L. (2006) An Update on the Diagnosis of Tuberculosis Infection. American Journal of Respiratory and Critical Care Medicine, 174, 736-742. https://doi.org/10.1164/rccm.200509-1516PP

[6] Scott, L., Da Silva, P., Boehme, C.C., Stevens, W. and Gilpin, C.M. (2017) Diagnosis of Opportunistic Infections: HIV Co-Infections-Tuberculosis. Current Opinion in HIV and AIDS, 12, 129-138. https://doi.org/10.1097/COH.0000000000000345

[7] Flores, L.L., Steingart, K.R., Dendukuri, N., Schiller, I., Minion, J., Pai, M., et al. (2011) Systematic Review and Meta-Analysis of Antigen Detection Tests for the Diagnosis of Tuberculosis. Clinical and Vaccine Immunology, 18, 1616-1627. https://doi.org/10.1128/CVI.05205-11

[8] Lawn, S.D. and Gupta-Wright, A. (2015) Detection of Lipoarabinomannan (LAM) in Urine Is Indicative of Disseminated TB with Renal Involvement in Patients Living with HIV and Advanced Immunodeficiency: Evidence and Implications. Transactions of the Royal Society of Tropical Medicine and Hygiene, 110, 180-185. https://doi.org/10.1093/trstmh/trw008

[9] Moore, D.A.J., Evans, C.A.W., Gilman, R.H., Sc, B., Coronel, J., et al. (2007) Microscopic-Observation Drug-Susceptibility Assay for the Diagnosis of TB. New England Journal of Medicine, 355, 1539-1550. https://doi.org/10.1056/NEJMoa055524

[10] World Health Organization (2014) Xpert MTB/RIF for People Living with HIV. World Health Organization, Geneva.

[11] Lawn, S.D., Brooks, S.V., Kranzer, K., Nicol, M.P., Whitelaw, A., Vogt, M., et al. (2011) Screening for HIV-Associated Tuberculosis and Rifampicin Resistance before Antiretroviral Therapy Using the Xpert MTB/RIF Assay: A Prospective Study. PLoS Medicine, 8, e1001067. https://doi.org/10.1371/journal.pmed.1001067

[12] Pai, M. (2013) Diagnosis of Pulmonary Tuberculosis: Recent Advances. Journal of Indian Medical Association, 111, 332-336.

https://www.e-trd.org/journal/view.php?doi=10.4046/trd.2015.78.2.64

[13] Salud, D.E. (2008) Demora en el Diagnóstico de Tuberculosis Pulmonar en una región de Colombia. Revista de Salud Pública, 10, 94-104. https://doi.org/10.1590/S0124-00642008000100009

[14] Budiarti, A., Nugraha, J., Dwi, A., Widodo, W., Program, I. and Airlangga, U. (2018) The Examination of ESAT-6, CFP-10 , MPT-64 Antigens of Mycobacterium tuberculosis in Urine of Pediatric Tuberculosis Patient with Immunochromatography. Folia Medica Indonesiana, 54, 195-199.

https://doi.org/10.20473/fmi.v54i3.10014

[15] Ramirez-Priego, P., Martens, D., Elamin, A.A., Soetaert, P., Van Roy, W., Vos, R., et al. (2018) Label-Free and Real-Time Detection of Tuberculosis in Human Urine Samples Using a Nanophotonic Point-of-Care Platform. ACS Sensors, 3, 2079-2086. https://doi.org/10.1021/acssensors.8b00393

[16] Reither, K., Saathoff, E., Jung, J., Minja, L.T., Kroidl, I., Saad, E., et al. (2009) Low Sensitivity of a Urine LAM-ELISA in the Diagnosis of Pulmonary Tuberculosis. BMC Infectious Diseases, 9, Article No. 141. https://doi.org/10.1186/1471-2334-9-141 http://bmcinfectdis.biomedcentral.com/articles/10.1186/1471-2334-9-141

[17] Tebianian, M., Mosavari, N., Taghizadeh, M. and Ebrahimi, S.M. (2016) Evaluation 
of Specific Antibodies against Mycobacterium tuberculosis Recombinant Antigens for Detection of Recent Infection. International Journal of Mycobacteriology, 5, S254. https://doi.org/10.1016/j.ijmyco.2016.11.011

[18] Zhang, X., Su, Z., Zhang, X., Hu, C., Yu, J., Gao, Q., et al. (2013) Generation of Mycobacterium Tuberculosis-Specific Recombinant Antigens and Evaluation of the Clinical Value of Antibody Detection for Serological Diagnosis of Pulmonary Tuberculosis. International Journal of Molecular Medicine, 31, 751-757. https://doi.org/10.3892/ijmm.2013.1254

[19] Araujo, Z., Giampietro, F., Bochichio, M.D.L.A., Palacios, A., Dinis, J., Isern, J., et al. (2013) Immunologic Evaluation and Validation Of methods Using Synthetic Peptides Derived from Mycobacterium tuberculosis for the Diagnosis of Tuberculosis Infection. Memórias do Instituto Oswaldo Cruz, 108, 131-139.

http://www.ncbi.nlm.nih.gov/pubmed/23579789 https://doi.org/10.1590/0074-0276108022013001

[20] Paris, L., Magni, R., Zaidi, F., Araujo, R., Saini, N., Harpole, M., et al. (2017) Urine Lipoarabinomannan Glycan in HIV-Negative Patients with Pulmonary Tuberculosis Correlates with Disease Severity. Science Translational Medicine, 9, eaal2807. https://doi.org/10.1126/scitranslmed.aal2807

[21] Magni, R., Espina, B.H., Liotta, L.A., Luchini, A. and Espina, V. (2014) Hydrogel Nanoparticle Harvesting of Plasma or Urine for Detecting Low Abundance Proteins. Journal of Visualized Experiments, No. 90, e51789.

https://doi.org/10.3791/51789

[22] Douglas, T.A., Tamburro, D., Fredolini, C., Espina, B.H., Lepene, B.S., Ilag, L., et al. (2011) Biomaterials the Use of Hydrogel Microparticles to Sequester and Concentrate Bacterial antigens in a Urine Test for Lyme Disease. Biomaterials, 32, 1157-1166. https://doi.org/10.1016/j.biomaterials.2010.10.004

[23] Castro-Sesquen, Y.E., Gilman, R.H., Mejia, C., Clark, D.E., Choi, J., Reimer-Mcatee, M.J., et al. (2016) Use of a Chagas Urine Nanoparticle Test (Chunap) to Correlate with Parasitemia Levels in T. cruzi/HIV Co-Infected Patients. PLoS Neglected Tropical Diseases, 10, e0004407. https://doi.org/10.1371/journal.pntd.0004407

[24] Castro-Sesquen, Y.E., Gilman, R.H., Galdos-Cardenas, G., Ferrufino, L., Sánchez, G., Valencia Ayala, E., et al. (2014) Use of a Novel Chagas Urine Nanoparticle Test (Chunap) for Diagnosis of Congenital Chagas Disease. PLoS Neglected Tropical Diseases, 8, e3211. https://doi.org/10.1371/journal.pntd.0003211

[25] Raveendran, R. and Wattal, C. (2016) Utility of Multiplex Real-Time PCR in the Diagnosis of Extrapulmonary Tuberculosis. The Brazilian Journal of Infectious Diseases, 20, 235-241. https://doi.org/10.1016/j.bjid.2016.01.006

[26] Mehta, P.K., Singh, N., Dharra, R., Dahiya, B., Sharma, S., Sheoran, A., et al. (2016) Diagnosis of Tuberculosis Based on the Detection of a Cocktail of Mycobacterial Antigen 85B, ESAT-6 and Cord Factor by Immuno-PCR. Journal of Microbiological Methods, 127, 24-27. https://doi.org/10.1016/j.mimet.2016.05.003

[27] Brock, M., Hanlon, D., Zhao, M. and Pollock, N.R. (2020) Detection of Mycobacterial Lipoarabinomannan in Serum for Diagnosis of Active Tuberculosis. Diagnostic Microbiology and Infectious Disease 96, Article ID: 114937.

https://doi.org/10.1016/j.diagmicrobio.2019.114937

[28] Broger, T., Sossen, B., Toit, E., Kerkhoff, A.D., Schutz, C., Reipold, E.I., et al. (2019) Articles Novel Lipoarabinomannan Point-of-Care Tuberculosis Test for People with HIV: A Diagnostic Accuracy Study. The Lancet Infectious Diseases, 19, 852-861. https://doi.org/10.1016/S1473-3099(19)30001-5 
[29] Kerkhoff, A.D., Sossen, B., Schutz, C., et al. (2020) Diagnostic Sensitivity of SILVAMP TB-LAM (FujiLAM) Point-of-Care Urine Assay for Extra-Pulmonary Tuberculosis in People Living with HIV. European Respiratory Journal, 55, Article ID: 1901259. https://doi.org/10.1183/13993003.01259-2019

[30] Hussain, M.M., Samir, T.M. and Azzazy, H.M.E. (2013) Unmodified Gold Nanoparticles for Direct and Rapid Detection of Mycobacterium tuberculosis Complex. Clinical Biochemistry, 46, 633-637.

https://doi.org/10.1016/j.clinbiochem.2012.12.020 